\title{
CONCENTRIC CIRCULAR MISFIT AND A CIRCULAR ARC CRACK IN AN INFINITE ISOTROPIC ELASTIC PLATE
}

\author{
R. D. BHARGAVA and RAM NARAYAN
}

(Received 6 March 1974)

(Revised 30 October 1975)

\begin{abstract}
A homogeneous isot ropic infinite elastic plate contains a circular cavity and a circular arc crack symmetrically situated about the $x$-axis. The cavity and crack are concentric but are of different radii. A circular inhomogeneity of radius slightly larger than that of the cavity is inserted into the cavity; thus generating a system of stresses in the outer material as well as in the inhomogeneity. The elastic field in the inhomogeneity and in the outer material outside the inhomogeneity is evaluated in this paper.
\end{abstract}

\section{Intreduction}

A number of problems [1-7] have been solved in elasticity theory on the stress distribution around a crack in an infinite flat plate subjected to either extensional or flexural loading at infinity. Muskhelishvili [8] has given simple method for solving two-dimensional circular arc crack problems. The problem of an arc crack around the boundary of a circular elastic inclusion was considered by A. H. England [9].

The study of inclusion problems was initiated by Frenkel [10], Mott and Nabarro [11]. A systematic investigation has been done by Eshelby [12] and by Jaswon and Bhargava [13] and others. The problem of the interaction between a crack and inclusion has been considered by Atkinson [14] and the effect of a circular inclusion on the stresses around a line crack in a sheet under tension by Tamate [15]. R. D. and R. R. Bhargava [16] have extended the method given by Tamate [15] to obtain the explicit solution to the problem of elastic circular inclusion in an infinite plate containing two straight cracks. 
In the present paper the problem of a circular misfit in an infinite plate containing a circular are crack is considered. The elastic properties of the two materials may differ from each other.

\section{Statement of the problem}

A homogeneous isotropic infinite elastic plate contains a circular cavity of radius $C$ and a concentric circular arc crack of radius $a(a>C)$ which is symmetrically situated about the $x$-axis. The crack subtends an angle $2 \alpha$ at its centre, the origin. The edges of the crack are free from external traction. A circular inhomogeneity of radius $(C+\varepsilon),(\varepsilon$ is of the order of the displacements admissible in elasticity theory), is inserted into the cavity and welded at the interface to avoid slipping. Because of the misfit, stresses will develop in the inhomogeneity and surrounding material. The problem is to evaluate the elastic field. The problem is considered as a plane strain problem and thus the $y$-axis is in the plane of the section (see Fig.1).

As is well-known [8], the stress components $P_{i,}(i, j=x, y)$ in cartesian coordinates or $(i, j=r, \theta)$ in polar coordinates and displacement components

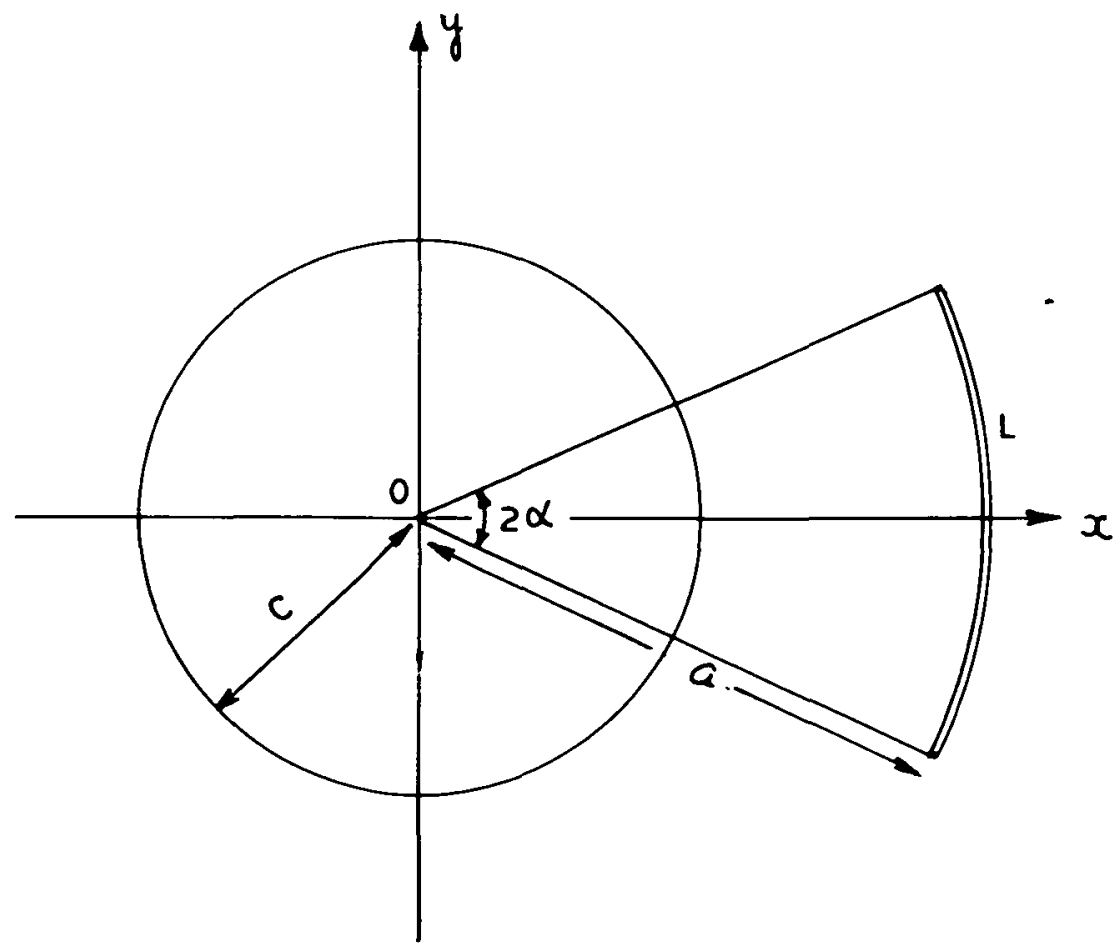

Fig. 1. Configuration and coordinate system 
$u_{1}(i=x, y$ or $i=r, \theta)$ are known if one can evaluate the complex potential functions $\phi(z)$ and $\psi(z)$ which are related to stresses as follows:

$$
\begin{aligned}
P_{x x}+P_{y y} & =2[\phi(z)+\overline{\phi(z)}], \\
P_{y y}-i P_{x y} & =\phi(z)+\overline{\phi(z)}+z \overline{\phi^{\prime}(z)}+\overline{\psi(z),} \\
2 \mu\left(u_{x, x}+i u_{y, x}\right) & =K \phi(z)-\phi(z)-z \phi^{\prime}(z)-\psi(z)
\end{aligned}
$$

or

$$
\begin{aligned}
P_{r}+P_{\theta \theta} & =2[\phi(z)+\overline{\phi(z)}] \\
P_{r}+i P_{r \theta} & =\phi(z)+\overline{\phi(z)}-\overline{z \phi^{\prime}(z)}-(\bar{z} / z) \overline{\psi(z)} \\
2 \mu \frac{\partial}{\partial \theta}\left[e^{i \theta}\left(u_{r}+i u_{\theta}\right)\right] & =i z\left[K \phi(z)-\overline{\phi(z)}+z \overline{\phi^{\prime}(z)}+(\bar{z} / z) \overline{\psi(z)}\right.
\end{aligned}
$$

where bars are used to denote conjugate complex quantities and subscripts following a comma stand for derivative with respect to the argument following it; $\mu$ is the shear modulus, $K=3-4 \sigma$ for plane strain and $K=(3-\sigma) /(1+\sigma)$ for generalized plane stress, $\sigma$ being Poisson's ratio.

If the stresses vanish at infinity, the functions $\phi(z)$ and $\psi(z)$ for large values of $z$ have the following forms:

$$
\begin{aligned}
& \phi(z)=\frac{a_{1}}{z^{2}}+\frac{a_{2}}{z^{3}}+\cdots, \\
& \psi(z)=\frac{b_{1}}{z^{2}}+\frac{b_{2}}{z^{3}}+\cdots,
\end{aligned}
$$

where $a_{k}$ 's and $b_{k}$ 's are constants.

It is convenient to introduce a new function $\Omega(z)$ by the relation

$$
\Omega(z)=\phi\left(\frac{a^{2}}{z}\right)-\frac{a^{2}}{z} \phi^{\prime}\left(\frac{a^{2}}{z}\right)-\frac{a^{2}}{z^{2}} \psi\left(\frac{a^{2}}{z}\right)
$$

which near the origin is of the order unity. By (5)

$$
\psi(z)=\frac{a^{2}}{z^{2}} \phi(z)-\frac{a^{2}}{z^{2}} \Omega\left(\frac{a^{2}}{z}\right)-\frac{a^{2}}{z} \phi^{\prime}(z) .
$$

Thus the equations in (2) may be written as

$$
\begin{aligned}
P_{r}+P_{\theta \theta} & =2[\phi(z)+\overline{\phi(z)}], \\
P_{r r}+i P_{r \theta} & =\phi(z)+\Omega\left(\frac{a^{2}}{z}\right)+\bar{z}\left(\frac{\bar{z}}{a^{2}}-\frac{1}{z}\right) \overline{\psi(z)}
\end{aligned}
$$




$$
2 \mu \frac{\partial}{\partial \theta}\left[e^{i \theta}\left(u_{r}+i u_{\theta}\right)\right]=i z K \phi(z)-\Omega\left(\frac{a^{2}}{z}\right)-\bar{z}\left(\frac{\bar{z}}{a^{2}}-\frac{1}{z}\right) \overline{\psi(z)} .
$$

For the present problem, two sets of functions, i.e. $\phi_{i}(z), \psi_{i}(z), \Omega_{1}(z)$ and $\phi_{m}(z), \psi_{m}(z), \Omega_{m}(z)$, are to be evaluated. The subscripts $i$ stand for the region of circular misfitting inhomogeneity and $m$ to the rest of the region containing the crack extending up to infinity.

\section{Boundary conditions}

The boundary conditions of the problem may be stated as follows:

(i) At infinity,

$$
\begin{aligned}
& \phi_{m}(z)=0\left(z^{-2}\right), \\
& \psi_{m}(z)=0\left(z^{-2}\right),
\end{aligned}
$$

hence near the origin,

$$
\Omega_{m}(z)=0(1)
$$

(ii) The rims of the crack $L$ are free from tractions. Therefore

$$
\left(P_{r r}^{ \pm}+i P_{r \theta}^{ \pm}\right)_{|\theta|<\alpha, r=a}=0 .
$$

This condition may be expressed in terms of $\phi_{m}(z)$ and $\Omega_{m}(z)$ as

$$
\begin{aligned}
& \phi_{m}^{+}(t)+\Omega_{m}^{-}(t)=0, \\
& \phi_{m}^{-}(t)+\Omega_{m}^{+}(t)=0,
\end{aligned}
$$

where $t$ is the coordinate of a point on the cut $L$ and superscripts $(+)$ and $(-)$ are used to denote the boundary values of the functions as $z$ tends to $t$ from the left and right regions of the crack (see Fig.1). By adding and subtracting, and using the obvious notation, equations (12) and (13) may be written as

$$
\begin{aligned}
& {\left[\phi_{m}(t)+\Omega_{m}(t)\right]^{+}+\left[\phi_{m}(t)+\Omega_{m}(t)\right]^{-}=0,} \\
& {\left[\phi_{m}(t)-\Omega_{m}(t)\right]^{+}-\left[\phi_{m}(t)-\Omega_{m}(t)\right]^{-}=0 .}
\end{aligned}
$$

(iii) On the common interface of the misfit and the outer material, the stresses $P_{r r}, P_{r e}$ are continuous and the discontinuity in displacement is as prescribed. This implies

$$
\left(P_{r}+i P_{r \theta}\right)_{m}=\left(P_{r r}+i P_{r \theta}\right)_{t}
$$

and

$$
\left(u_{r}+i u_{\theta}\right)_{m}-\left(u_{r}+i u_{\theta}\right)_{\imath}=\varepsilon \text {. }
$$


From equations (14) and (15), which is the Hilbert problem, we can construct the function $\phi_{m}(z), \Omega_{m}(z)$ analytic in the entire region cut along $L$. Taking into account the fact that $\phi_{m}(z)$ and $\Omega_{m}(z)$ could have poles of various orders at the origin and still satisfy the conditions at infinity as well as along the rims of the crack, $\phi_{m}(z), \Omega_{m}(z)$ may be written as

$$
\begin{aligned}
\Phi_{m}(z)= & \frac{1}{2} \sum_{n=2}^{\infty} A_{-n}\left(\frac{a}{z}\right)^{n}+\sum_{n=0}^{\infty} A_{n}\left(\frac{z}{a}\right)^{n} \\
& +\frac{1}{X(z)}\left\{\sum_{n=1}^{\infty} B_{-n}\left(\frac{a}{z}\right)^{n}+\sum_{n=0}^{\infty} B_{n}\left(\frac{z}{a}\right)^{n}\right\}, \\
\Omega_{m}(z)= & \frac{1}{2}\left[-\sum_{n=2}^{\infty} A_{-n}\left(\frac{a}{z}\right)^{n}-\sum_{n=0}^{\infty} A_{n}\left(\frac{z}{a}\right)^{n}\right. \\
& \left.+\frac{1}{X(z)}\left\{\sum_{n=1}^{\infty} B_{-n}\left(\frac{a}{z}\right)^{n}+\sum_{n=0}^{\infty} B_{n}\left(\frac{z}{a}\right)^{n}\right\}\right] .
\end{aligned}
$$

Note that in the summation symbol in the first terms of the right sides of $\Phi_{m}(z)$ and $\Omega_{m}(z)$ in equation (18), $A_{-1}$ has been set equal to zero. This is because $\operatorname{Im}\left(A_{-1}\right)$ is directly proportional to the resultant external force on the crack and internal boundary of the matrix. Note that the external force on the crack is already taken to be zero. As regards the internal circular boundary of the elastic plate (circular hole), the resultant force on it is proportional to coefficient of $\operatorname{Im}\left(A_{-1}\right)$ in (18), and this is clearly zero because the inclusion does not give any resultant force on matrix. $\operatorname{Re}\left(A_{-1}\right)$ is zero because of single-valuedness of the displacements. ${ }^{*}$ Also $A_{n}, A_{-n}, B_{n}$ and $B_{-n}$ are unknown constants and $X(z)$ denotes one of the branches of

$$
X(z)=\left(\frac{z}{a}-e^{-i \alpha}\right)^{1 / 2}\left(\frac{z}{a}-e^{i \alpha}\right)^{1 / 2}
$$

which is single-valued in the entire plane cut along $L$. We take that branch for which $X(z) \rightarrow(z / a)$ for $z \rightarrow \infty$. We now put

$$
\left(1-z_{1} e^{-i \alpha}\right)^{-1 / 2}\left(1-z_{1} e^{i \alpha}\right)^{-1 / 2}=\sum_{n=0}^{\infty} P_{-(n+1)} z_{i}^{n}
$$

and write

$$
P_{n}=-p_{-(n+1),}, \quad(n \geqq 0) .
$$

Using the expression for $\phi_{m}(z)$ in (18), and the condition at infinity in (9), we get

* The proof of this was given by the referee in a private communication to the first author and is given in the Appendix. 


$$
\begin{gathered}
A_{n}=-\sum_{k=1}^{\infty} p_{-k} B_{n+k}, \quad(n \geqq 0), \\
\sum_{k=1}^{\infty} p_{-k} B_{k-1}=0 .
\end{gathered}
$$

Also by the expression for $\Omega_{m}(z)$ in (18) and the condition (10), we obtain the following equations:

$$
\begin{gathered}
A_{-n}=\sum_{k=0}^{\infty} p_{k} B_{-(k+n), \quad(n \geqq 2),} \\
\sum_{k=0}^{\infty} p_{k} B_{-(k+1)}=0 .
\end{gathered}
$$

The function $\phi_{m}(z)$ in (18) and the corresponding function $\psi_{m}(z)$ which is derived from (6) may be expanded in the region $0<|z|<a$ in Laurent series, using (18a) and (18b) as follows:

$$
\begin{aligned}
& \phi_{m}(z)=\frac{1}{2} \sum_{n=-\infty}^{\infty} E_{n}\left(\frac{z}{a}\right)^{n}, \\
& \psi_{m}(z)=\frac{1}{2} \sum_{n=-\infty}^{\infty} F_{n}\left(\frac{z}{a}\right)^{n}
\end{aligned}
$$

where

$$
\begin{aligned}
E_{-1} & =0, \\
E_{-n} & =2 \sum_{k=0}^{\infty} p_{k} B_{-(k+n)}, \quad(n \geqq 2), \\
E_{n} & =\sum_{k=0}^{\infty} p_{k} B_{n-k}-\sum_{k=1}^{\infty} p_{-k} B_{n+k}, \quad(n \geqq 0),
\end{aligned}
$$

and

$$
\begin{aligned}
F_{-1}= & 0 \\
F_{-n}= & (n-1) E_{-(n-2)}-2 \sum_{k=1}^{\infty} p_{-k} B_{n+k-2}, \quad(n \geqq 2), \\
F_{n}= & (n+1)\left[\sum_{k=1}^{\infty} p_{-k} B_{n+k+2}-\sum_{k=0}^{\infty} p_{k} B_{n+2-k}\right] \\
& +\sum_{k=0}^{\infty} p_{k} \bar{B}_{-(k+n+2)}-\sum_{k=1}^{\infty} p_{-k} \bar{B}_{(n-k+2)}, \quad(n \geqq 0) .
\end{aligned}
$$

Reverting to the inclusion, it is observed that complex potentials $\phi_{1}(z)$ and $\psi_{1}(z)$ are holomorphic in the region $|z| \leqq C$. Hence by Taylor's series 


$$
\begin{aligned}
& \phi_{i}(z)=\frac{1}{2} \sum_{n=0}^{\infty} G_{n}\left(\frac{z}{C}\right)^{n}, \\
& \psi_{i}(z)=\frac{1}{2} \sum_{n=0}^{\infty} H_{n}\left(\frac{z}{C}\right)^{n},
\end{aligned}
$$

where the coefficients $G_{n}$ and $H_{n}$ are unknown and are to be determined by the conditions along the bonded edge $|z|=C$.

At this stage it is necessary to translate the conditions (16) and (17) in terms of $\phi(z)$ and $\psi(z)$. The condition (16) with the help of (2) may be written as

$$
\begin{aligned}
\phi_{m} & \left(C e^{i \theta}\right)+\bar{\phi}_{m}\left(C e^{i \theta}\right)-C e^{-i \theta} \bar{\phi}^{\prime}\left(C e^{-i \theta}\right)-e^{-2 t \theta} \bar{\psi}_{m}\left(C e^{-i \theta}\right) \\
& =\phi_{i}\left(C e^{i \theta}\right)+\bar{\phi}_{i}\left(C e^{-i \theta}\right)-C e^{-i \theta} \bar{\phi}_{1}^{\prime}\left(C e^{-i \theta}\right)-e^{-2 i \theta} \bar{\psi}_{1}\left(C e^{-i \theta}\right) .
\end{aligned}
$$

Similarly using (17), with help of (2) may be written as,

$$
\begin{gathered}
\frac{\mu_{i}}{\mu_{m}}\left\{K_{m} \phi_{m}\left(C e^{i \theta}\right)-\bar{\phi}_{m}\left(C e^{-1 \theta}\right)+C e^{-i \theta} \bar{\phi}_{m}^{\prime}\left(C e^{-1 \theta}\right)+e^{-2 \theta \theta} \bar{\psi}_{m}\left(C e^{-i \theta}\right)\right\} \\
-\left\{K_{1} \phi_{1}\left(C e^{i \theta}\right)-\bar{\phi}_{1}\left(C e^{-1 \theta}\right)+C e^{-i \theta} \bar{\phi}_{1}^{\prime}\left(C e^{-i \theta}\right)+e^{-2 i \theta} \bar{\psi}_{i}\left(C e^{-i \theta}\right)\right\}=\frac{2 \varepsilon \mu_{i}}{C} .
\end{gathered}
$$

We now substitute the values of $\phi_{m}(z), \psi_{m}(z)$ from (21) and $\phi_{i}(z)$ and $\psi_{1}(z)$ from (24) into the above equations. Equating the coefficients of various powers of $e^{i \theta}$ on both sides; the following equations are obtained.

$$
\begin{gathered}
E_{0}+\bar{E}_{0}-\left(\frac{a}{C}\right)^{2} \bar{F}_{-2}=G_{0}+\bar{G}_{0}, \\
\frac{\mu_{i}}{\mu_{m}}\left(K_{m} E_{0}-\bar{E}_{0}\right)+\frac{\mu_{i}}{\mu_{m}}\left(\frac{a}{C}\right)^{2} \bar{F}_{-2}=\frac{4 \varepsilon \mu_{i}}{C}+K_{i} G_{0}-G_{0} \\
E_{n}\left(\frac{C}{a}\right)^{n}+(n+1)\left(\frac{a}{C}\right)^{n} \bar{E}_{-n}-\left(\frac{a}{C}\right)^{n+2} F_{-(n+2)}=G_{n}, \\
\frac{\mu_{i} K_{m}}{\mu_{m}}\left(\frac{C}{a}\right)^{n} E_{n}-\frac{\mu_{i}}{\mu_{m}}(n+1) \bar{E}_{-n}\left(\frac{a}{C}\right)^{n}+\frac{\mu_{1}}{\mu_{m}} \bar{F}_{-(n+2)}\left(\frac{a}{C}\right)^{n+2}=K_{i} G_{n}, \\
\left.\left(\frac{a}{C}\right)^{n} E_{-n}-(n-1)\left(\frac{C}{a}\right)^{n} \bar{E}_{n}-\left(\frac{C}{a}\right)^{n-2} \bar{F}_{n-2}=-(n-1), \cdots\right), \\
\left(\frac{a}{C}\right)^{n} \frac{\mu_{i} K_{m}}{\mu_{m}} E_{-n}+(n-1)\left(\frac{C}{a}\right)^{n} \frac{\mu_{1}}{\mu_{m}} E_{n}+\left(\frac{C}{a}\right)^{n-2} \frac{\mu_{i}}{\mu_{m}} F_{n-2}=(n-1) \bar{G}_{n-2}+\bar{H}_{n-2}, \\
(n=2,3, \cdots) .
\end{gathered}
$$

Eliminating $G_{n}$ and $H_{n-2}$ from equations (27), 


$$
\begin{array}{r}
E_{-n}=-\frac{\left(\frac{\mu_{i}}{\mu_{m}}\right)-1}{\frac{\mu_{1} K_{m}}{\mu_{m}}+1}\left\{(n-1)\left(\frac{C}{a}\right)^{2 n} E_{n}+\left(\frac{C}{a}\right)^{2 n-2} \bar{F}_{n-2}\right\}, \\
(n=2,3, \cdots) .
\end{array}
$$

From (25) and (26), we get

$$
\begin{aligned}
& F_{-2}=\frac{\dot{8} \mu_{i} \varepsilon}{\left(\frac{2 \mu_{1}}{\mu_{m}}+K_{t}-1\right) C}\left(\frac{C}{a}\right)^{2}-\frac{\left(\frac{\mu_{i} K_{m}}{\mu_{m}}\right)-K_{i}-\left(\frac{\mu_{i}}{\mu_{m}}\right)+1}{\left(\frac{2 \mu_{i}}{\mu_{m}}\right)+K_{1}-1}\left(E_{0}+\bar{E}_{0}\right)\left(\frac{C}{a}\right) \\
& F_{-(n+2)}=(n+1)\left(\frac{C}{a}\right)^{2} E_{-n}-\frac{\left(\frac{\mu_{1} K_{m}}{\mu_{m}}\right)-K_{t}}{\left(\frac{\mu_{1}}{\mu_{m}}\right)+K_{t}} \bar{E}_{n}\left(\frac{C}{a}\right)^{2 n+2} \quad(n=1,2, \cdots) .
\end{aligned}
$$

Equations (25), (26) and (27) are solved for $G_{n}$ and $H_{n}$ as follows:

$$
\begin{aligned}
& G_{n}= \frac{\left(\frac{\mu_{1} K_{m}}{\mu_{m}}\right)+1}{K_{1}+1}\left(\frac{C}{a}\right)^{n} E_{n}+\frac{1-\left(\frac{\mu_{1}}{\mu_{m}}\right)}{K_{t}+1}(n+1)\left(\frac{a}{C}\right)^{n} \bar{E}_{-n} \\
&+\frac{\left(\frac{\mu_{1}}{\mu_{m}}\right)-1}{K_{1}+1}\left(\frac{a}{C}\right)^{n+2} \bar{F}_{-(n+2)}-\frac{4 \varepsilon \mu_{i}}{C\left(1+K_{t}\right)} \delta_{0, n} \\
& H_{n}=(n+1)\left(\frac{a}{C}\right)^{n+4} \bar{F}_{-(n+4)}+\left(\frac{C}{a}\right)^{n} F_{n}-(n+2)^{2}\left(\frac{a}{C}\right)^{n+2} \bar{E}_{-(n+2)} \\
& \quad(n=0,1,2, \cdots) .
\end{aligned}
$$

Substituting equations (22), (23) to (28) and (29), we obtain the following infinite set of linear equations for $B_{n}$ and $B_{-n}$.

$$
\begin{aligned}
& \sum_{k=0}^{\infty} p_{k} B_{-(k+1)}=0 \\
& \sum_{k=0}^{\infty} p_{k} B_{-(k+n)}=\frac{1}{2} \frac{\left(\frac{\mu_{i}}{\mu_{m}}\right)-1}{\left(\frac{\mu_{t} K_{m}}{\mu_{m}}\right)+1}\left(\frac{C}{a}\right)^{2 n-2} \times \\
& {\left[(n-1)\left(1-\frac{C^{2}}{a^{2}}\right)\left(\sum_{k=0}^{\infty} p_{k} \bar{B}_{n-k}-\sum_{k=1} p_{-k} \bar{B}_{k+n}\right)+\sum_{k=1}^{\infty} p_{-k} B_{k-n}-\sum_{k=0}^{\infty} p_{k} B_{-(k+n)}\right] .}
\end{aligned}
$$




$$
\begin{aligned}
\sum_{k=1}^{\infty} p_{-k} \bar{B}_{k-1}= & 0, \\
\sum_{k=1}^{\infty} p_{-k} \bar{B}_{k}= & \frac{1}{2}\left(\sum_{k=0}^{\infty} p_{k} B_{-k}-\sum_{k=1}^{\infty} p_{-k} B_{k}\right)-\frac{4 \varepsilon \mu_{i}}{\left(2 \frac{\mu_{i}}{\mu_{m}}+K_{i}-1\right) C}\left(\frac{C}{a}\right)^{2} \\
& +\frac{1}{2} \frac{\left(\frac{\mu_{1} K_{m}}{\mu_{m}}\right)-K_{i}-\left(\frac{\mu_{i}}{\mu_{m}}\right)+1}{2\left(\frac{\mu_{i}}{\mu_{m}}\right)+K_{t}-1}\left(\frac{C}{a}\right)^{2}\left[\sum_{k=0}^{\infty} p_{k} B_{-k}\right. \\
& \left.-\sum_{k=1}^{\infty} p_{-k} B_{k}+\sum_{k=0}^{\infty} p_{k} \bar{B}_{-k}-\sum_{k=1}^{\infty} \dot{p}_{-k} \bar{B}_{k}\right], \\
\sum_{k=1}^{\infty} p_{-k} \bar{B}_{k+n}= & (n+1)\left(1-\frac{C^{2}}{a^{2}}\right) \sum_{k=0}^{\infty} p_{k} B{ }_{-(n+k)} \\
& +\frac{1}{2} \frac{\left(\frac{\mu_{i} K_{m}}{\mu_{m}}\right)-K_{1}}{\left(\frac{\mu_{1}}{\mu_{m}}\right)+K_{i}} \cdot\left(\frac{C}{a}\right)^{2 n+2}\left(\sum_{k=0}^{\infty} p_{k} \bar{B}_{n-k}-\sum_{k=1}^{\infty} p_{-k} \bar{B}_{k+n}\right)
\end{aligned}
$$

Equations (31) and (32) are the infinite set of linear equations in which $K_{\text {, }}$ $K_{m}, \mu_{t}, \mu_{m}, p_{-k}, p_{k} ;(C / a)$ and $\varepsilon$ are known. These sets determine the values of unknown constants $B_{n}$ and $B_{-n}$. After the determination of $B_{n}$ and $B_{-n}$, the coefficients $A_{n}, A_{-n}$ are determined by (19) and (20). These values when substituted in (18) determine the potential function $\phi_{m}(z)$ and $\Omega_{m}(z) ; \psi_{m}(z)$ may be obtained from (6). The stresses in the matrix are determined by using these values of $\phi_{m}(z), \psi_{m}(z)$ in (18). Alternatively one may determine the values of $E_{n}, E_{-n}$ by substituting the above known values of $B_{n}$ and $B_{-n}$ in (22). Substituting these values of $B_{n}, B_{-n}$, and $E_{-n}$ one may obtain $F_{n}$ and $F_{-n}$ from (23). These values when substituted in (21) determine $\phi_{m}(z), \psi_{m}(z)$. These will determine the stress field in the matrix.

As regards misfitting inhomogeneity, one may determine the values of $G_{n}$ and $H_{n}$ from (30) by substituting the values of $E_{n}, E_{-n}, F_{n}$ and $F_{-n}$ as indicated above. These values of $G_{n}$ and $H_{n}$ when substituted in (24) determine $\phi_{i}(z)$ and $\psi_{i}(z)$ which determine the stress and displacement field in the inhomogeneity.

It is of some interest to note the stress intensity factor at the crack tips. For this purpose we use the definition given by Sih, Paris and Erdogan [17]. In this paper the stress intensity factor is defined by $\left(K_{1}-i K_{2}\right)$. Using the above definition, the stress-intensity factor for the present problem comes out to be 


$$
\begin{aligned}
K_{1}= & \sqrt{\frac{a}{\sin \alpha}}\left[\left\{B_{0}^{(i)}+\sum_{k=1}^{\infty}\left(\left(B_{n}^{(r)}-B_{-n}^{(r)}\right) \sin n \alpha+\left(B_{n}^{(i)}+B_{-n}^{(i)}\right) \cos n \alpha\right)\right\} \cos \frac{\alpha}{2}\right. \\
& \left.-\left\{B_{0}^{(r)}+\sum_{n=1}^{\infty}\left(\left(B_{n}^{(r)}+B_{-n}^{(r)}\right) \cos n \alpha-\left(B_{n}^{(1)}-B_{-n}^{(i)}\right) \sin n \alpha\right)\right\} \sin \frac{\alpha}{2}\right] . \\
K_{2}= & \sqrt{\frac{a}{\sin \alpha}}\left[\left\{B_{0}^{(r)}+\sum_{n=1}^{\infty}\left(\left(B_{n}^{(r)}+B_{-n}^{(r)}\right) \cos n \alpha-\left(B_{n}^{(i)}-B_{-n}^{(i)}\right) \sin n \alpha\right)\right\} \cos \frac{\alpha}{2}\right. \\
& \left.+\left\{B_{0}^{(i)}+\sum_{n=1}^{\infty}\left(\left(B_{n}^{(r)}-B_{-n}^{(r)}\right) \sin n \alpha+\left(B_{n}^{(1)}+B_{-n}^{(i)}\right) \cos n \alpha\right)\right\} \sin \frac{\alpha}{2}\right],
\end{aligned}
$$

where the values of $B_{n}$ 's are to be obtained as stated above. The symbols $B_{n}^{(r)}$, $B_{n}^{(1)}$ denote respectively the real and imaginary part of $B_{n}$ 's.

To obtain explicit values for some cases, one may solve numerically the equations (31) and (32). For the purpose of illustration, two materials were chosen, some kind of brass and of steel for which shear moduli are $3.66 \times 100$ $\mathrm{gm} / \mathrm{cm}^{2}$ and $8.19 \times 10 \mathrm{gm} / \mathrm{cm}^{2}$ and Poisson's ratio 0.327 and 0.310 respectively. These figures have been taken from [18]. Two cases were considered when $2 \alpha=\pi / 18$ and $\pi / 9$. Equations (31) and (32) were solved by the standard iteration procedure. Values of $B_{n}$ 's were found successively. It turned out that after 16 iterations, the values of 15 unknowns $\left(B_{1}, B_{2}, \cdots, B_{7}\right.$; $\left.B_{-1}, B_{-2}, \cdots, B_{-8}\right)$ were identical up to the 5 th place of decimal. Moreover the values of $B_{6}, B_{7}, B_{-7}, B_{-8}$ were zero up to 5 places of decimals. It was decided therefore that this will give sufficiently good results. Two checks are still needed, one to find the value of $P_{r}, P_{r \theta}$ at the boundary of the crack, which should be zero, and those of $P_{r}, P_{r \theta}$ at the interface which should be continuous and also that the discontinuity in displacement should be as prescribed.

As regards the crack boundary, from equations (7)

$$
\left(P_{n}^{+}+i P_{r \theta}^{+}\right)_{|\theta|<\alpha, r=a}=\phi^{+}(t)+\Omega^{-}(t) .
$$

Substituting the values of $\phi(t)$ and $\Omega(t)$ etc. and retaining equal number of terms, it may be directly seen, that $\phi^{+}(t)+\Omega^{-}(t)$ is identically zero. As regards the stress at $r=a$ and $|\theta|>\alpha$, the substitution of the values of $B_{n}$ 's gave the values of $P_{r r}$ and $P_{r \theta}$ for matrix and inclusion side which differed only in the fourth place. This was true for displacements also.

Graphs for stress intensity factor have been drawn in Figures 2 (i), 2 (ii), 2 (iii) for 3 cases namely when (i) the inhomogeneity is of brass, matrix of steel (ii) inhomogeneity of steel and matrix of brass and (iii) when inhomogeneity and matrix are both of steel. The radius of inhomogeneity is $(1+\varepsilon)$ and the 


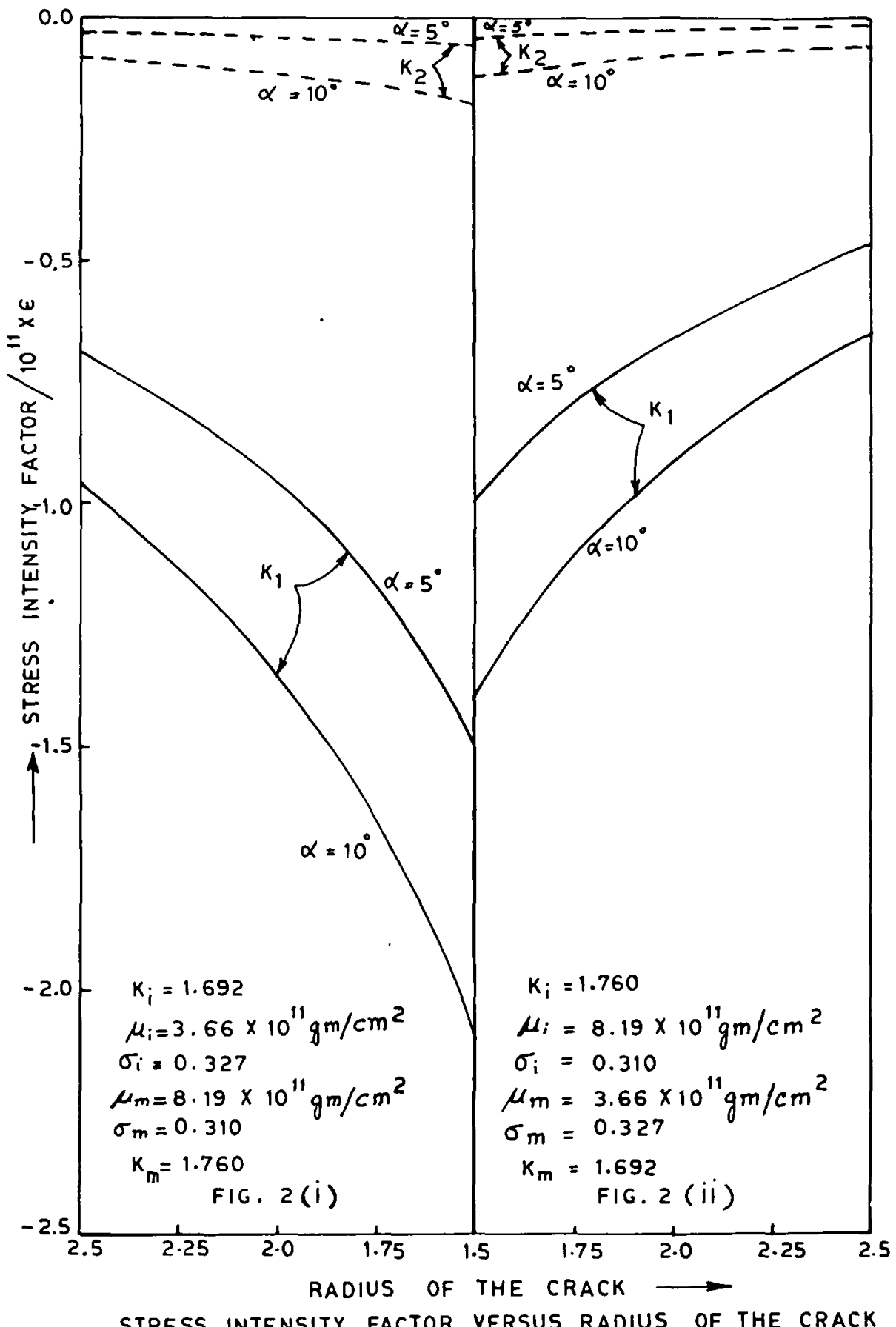




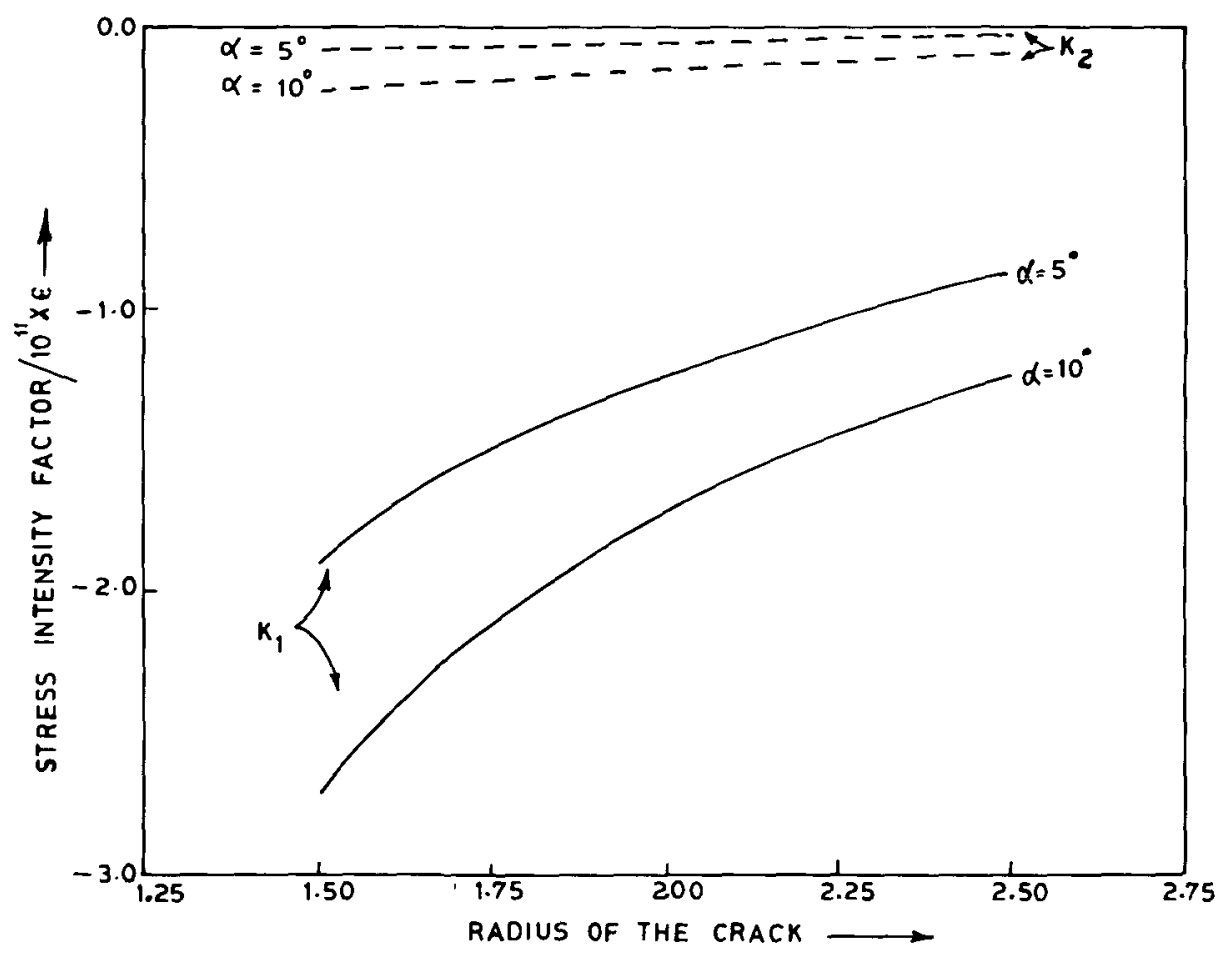

FIG. 2 (iii) STRESS INTENSITY FACTOR VERSUS RADIUS OF THE CRACK

crack radii are changing. The full lines denote the stress intensity factor $K_{1}$ and the broken lines $K_{2}$.

Note that we have taken $\varepsilon$ to be positive, whence the stress intensity factor came out to be negative. This indicates that the crack would close. In fact the above analysis is valid only for negative values of $\varepsilon$. This is also confirmed on physical grounds.

\section{Acknowledgement}

The authors are grateful to the referee for his suggestions which have improved the paper.

\section{Appendix}

The displacement components $u_{x}+i u_{y}$ are related to complex potentials $\varphi(z), \psi(z)$ by the relations 


$$
2 \mu\left(u_{x}+i u_{y}\right)=K \varphi(z)-\overline{z \varphi^{\prime}(z)}-\overline{\psi(z)}
$$

where $\varphi^{\prime}(z)=\Phi(z)$ and $\psi^{\prime}(z)=\Psi(z)$. Substituting $\Phi_{m}(z)$ and $\Psi_{m}(z)$ for $\Phi(z)$ and $\Psi(z)$ and putting the values of $\Phi_{m}(z)$ and $\Psi_{m}(z)$ from (21), it is seen that $u_{x}+i u_{y}$ is single-valued only if

$$
K E_{-1}-F_{-1}=0 \text {. }
$$

Similarly the resultant traction $X+i Y$ on the circular boundary from $A$ to $B$ is given by

$$
X+i Y=\int_{A B}\left(P_{n x}+i P_{n y}\right) d s=-i\left[\varphi(z)+\overline{z \varphi^{\prime}(z)}+\overline{\psi(z)}\right]_{A}^{B}
$$

where [ $]_{A}^{B}$ denotes the increase undergone by the expression in the bracket as the point $z$ passes along the arc from $A$ to $B$. Taking the closed circular curve, if the resultant traction is zero,

$$
E_{-1}+F_{-1}=0 \text {. }
$$

From (A.1) and (A.2)

$$
E_{-1}=0, \quad F_{-1}=0 .
$$

Now if in (18), the term containing $A_{-1}$ is retained, equations (19) and (20), would be

$$
A_{-1}+\sum_{1}^{\infty} p_{-1} B_{k-1}=0 \text { and } A_{-1}-\sum_{0}^{\infty} p_{k-(k+1)}=0
$$

and then in (22),

$$
E_{-1}=A_{-1}-2 \sum_{0}^{\infty} p_{k} B_{-(k+1)}=-A_{1}
$$

whence $\boldsymbol{A}_{-1} \equiv 0$.

\section{References}

[1] M. L. Williams, J. Appl. Mech., 24 (1957).

[2] M. L. Williams, J. Appl. Mech., 28 (1961).

[3] F. Erdogan, J. Appl. Mech., 30 (1963).

[4] G. C. Sih and J. R. Rice, J. Appl. Mech., 31 (1964).

[5] O. Tamate, Technology Reports, Tohoku University, 30 (1965).

[6] W. G. Knauss, J. Appl. Mech., 33 (1966).

[7] O. Tamate, Ing. Archiv., 35 (1967).

[8] N. I. Muskhelishvili, Some basic problems of the mathematical theory of elasticity, ( $P$. Noordhoff, Groningen, 1953).

[9] A. H. England, J. Appl. Mech., 33 (1966). 
[10] J. Frenkel, Kinetic theory of liquids, (Oxford 1946).

[11] N. F. Mott and F. R. N. Nabarro, Proc. Phys. Soc. 52 (1940).

[12] J. D. Eshelby, Proc. Roy. Soc. London, Ser. A241 (1957) ibid., 252 (1959).

[13] M. A. Jaswon and R. D. Bhargava, Proc. Camb. Phil. Soc. 57 (1961).

[14] C. Atkinson, Int. J. Engg. Sc. 4 (1969).

[15] O. Tamate, Int. J. Frac. Mech., 4 (1968).

[16] R. D. Bhargava and R. R. Bhargava, Int. J. Engg. Sc. 11 (1973).

[17] G. C. Sih, P. C. Paris and F. Erdogan, J. Appl. Mech. 29 (1962).

[18] A. E. H. Love, A treatise on the mathematical theory of elasticity, (Cambridge, 1952).

Department of Mathematics,

Indian Institute of Technology,

Bombay -76 ,

India. 